\title{
ITERATED LIMITS AND THE CENTRAL LIMIT THEOREM FOR DEPENDENT VARIABLES ${ }^{1}$
}

GEORGE MARSAGLIA

1. Introduction. $\S 2$ of this paper gives some results on iterated limits which may be considered generalizations of well-known results [1, p. 254]. $\S 3$ applies these results to give easy proofs of some central limit theorems for $m$-dependent variables. Compare Theorem 2 in [2] and Theorem 1 in [3].

2. Iterated probability limits. Here, we use the strong sense of an iterated limit: for constants $a_{i j}, i, j=1,2, \cdots, \lim _{j} \lim _{i} a_{i j}=a$ means

$$
\lim _{j \rightarrow \infty}\left(\limsup _{i \rightarrow \infty}\left|a_{i j}-a\right|\right)=0 .
$$

We note that (1) holds if, and only if, for each $\epsilon>0$ there exist integers $M, N_{1}, N_{2}, \cdots$ such that if the pair $(i, j)$ satisfies $j>M$, $i>N_{j}$, then $\left|a_{i j}-a\right|<\epsilon$.

Definition 1. Let $f, f_{i j}, i, j=1,2, \cdots$, be random variables. Then

$$
\text { plim } \operatorname{plim}_{i} f_{i j}=f
$$

means, for every $\epsilon>0$,

$$
\lim _{j} \lim _{i} P\left(\left|f_{i j}-f\right|>\epsilon\right)=0 .
$$

Theorem 1. Let $h_{i j}, g_{i j}, i, j=1,2, \cdots$ be random variables. Let $G$ be a function such that at each of its continuity points $x$

$$
\lim _{\mathfrak{l}} \lim _{i} P\left(g_{i j} \leqq x\right)=G(x) \text {, }
$$

and suppose

$$
\operatorname{plim}_{\mathfrak{j}} \operatorname{plim}_{i} h_{i j}=0
$$

Then

$$
\lim _{j} \lim _{i} P\left(g_{i j}+h_{i j} \leqq x\right)=G(x) .
$$

Let $\epsilon=8 \delta>0$ and a continuity point $x$ of $G$ be given. We shall exhibit integers $M, N_{1}, N_{2}, \cdots$ such that

Received by the editors April 19, 1954.

${ }^{1}$ Work sponsored by the Office of Naval Research under Contract No. N7-onr28402. 


$$
\left|P\left(g_{i j}+h_{i j} \leqq x\right)-G(x)\right|<\epsilon
$$

if $j>M$ and $i>N_{j}$. First, choose $\beta$ so that $G$ is continuous at $x+\beta$, at $x-\beta$, and so that

$$
|G(x+\beta)-G(x-\beta)|<\delta .
$$

Then choose $M, N_{1}, N_{2}, \cdots$ so that, simultaneously,

$$
\begin{array}{r}
P\left(\left|h_{i j}\right|>\beta\right)<\delta, \\
\left|P\left(g_{i j} \leqq x\right)-G(x)\right|<\delta, \\
\left|P\left(g_{i j} \leqq x-\beta\right)-G(x-\beta)\right|<\delta, \\
\left|P\left(g_{i j} \leqq x+\beta\right)-G(x+\beta)\right|<\delta
\end{array}
$$

whenever $(i, j)$ satisfies $j>M$ and $i>N_{j}$. Then for such a pair $(i, j)$, let $F(x)=P\left(g_{i j}+h_{i j} \leqq x\right), H(x, \beta)=P\left(g_{i j}+h_{i j} \leqq x,\left|h_{i j}\right| \leqq \beta\right), L(x, \beta)$ $=P\left(g_{i j} \leqq x,\left|h_{i j}\right| \leqq \beta\right)$, and $Q(x)=P\left(g_{i j} \leqq x\right)$. We have

$$
\begin{gathered}
|F(x)-G(x)| \leqq|F(x)-H(x, \beta)|+|H(x, \beta)-L(x, \beta)| \\
+|L(x, \beta)-Q(x)|+|Q(x)-G(x)| .
\end{gathered}
$$

Now by (4) and (5), each of the terms on the right except the second is bounded by $\delta$, and since $L(x-\beta, \beta) \leqq H(x, \beta) \leqq L(x+\beta, \beta)$ and $L(x-\beta, \beta) \leqq L(x, \beta) \leqq L(x+\beta, \beta)$,

$$
|H(x, \beta)-L(x, \beta)| \leqq|L(x+\beta, \beta)-L(x-\beta, \beta)|<5 \delta
$$

by (4), (7), (3), and (6), since

$$
\begin{aligned}
\mid L(x+\beta, \beta) & -L(x-\beta, \beta) \mid \\
\leqq & |L(x+\beta, \beta)-Q(x+\beta)|+|Q(x+\beta)-G(x+\beta)| \\
& +|G(x+\beta)-G(x-\beta)|+|G(x-\beta)-Q(x-\beta)| \\
& +|Q(x-\beta)-L(x-\beta, \beta)| .
\end{aligned}
$$

Hence

$$
|F(x)-G(x)|<8 \delta=\epsilon,
$$

which is condition (2).

THEOREM 2. Under the conditions of Theorem 1, if there exist constants $a_{i j}$ such that $\lim _{j} \lim _{i} a_{i j}=a>0$, and if $G$ is continuous at $x / a$, then $\lim _{j} \lim _{i} P\left(a_{i j} g_{i j} \leqq x\right)=G(x / a)$.

Using the artifice, for suitable $i, j, \gamma$ 


$$
\begin{aligned}
&\left|P\left(g_{i j} \leqq \frac{x}{a_{i j}}\right)-G\left(\frac{x}{a}\right)\right| \leqq \\
&\left|P\left(g_{i j} \leqq \frac{x}{a_{i j}}\right)-P\left(g_{i j} \leqq \frac{x}{a}\right)\right| \\
& \quad+\left|P\left(g_{i j} \leqq \frac{x}{a}\right)-G\left(\frac{x}{a}\right)\right|, \\
&\left|P\left(g_{i j} \leqq \frac{x}{a_{i j}}\right)-P\left(g_{i j} \leqq \frac{x}{a}\right)\right| \\
& \leqq\left|P\left(g_{i j} \leqq \frac{x}{a-\gamma}\right)-P\left(g_{i j} \leqq \frac{x}{a+\gamma}\right)\right|,
\end{aligned}
$$

the proof is routine. The details are omitted.

3. Applications to partitioned sequences of $m$-dependent random variables. Let $x_{1}, x_{2}, \cdots$ be an $m$-dependent sequence of random variables with zero means. For each pair $(n, k)$ with $2 m<k \leqq n$, define

$$
\begin{array}{rlrl}
y_{i} & =x_{i k-k+1}+\cdots+x_{i k-m}, & i=1,2, \cdots, \\
g_{n k} & =\sum_{1}^{[n / k]} y_{i}, & t_{n k}^{2} & =E\left(g_{n k}^{2}\right), \\
s_{n}^{2} & =E\left(x_{1}+\cdots+x_{n}\right)^{2}, & h_{n k} & =\frac{1}{s_{n}}\left(\sum_{1}^{n} x_{i}-g_{n k}\right) .
\end{array}
$$

Since we shall be dealing with $\lim _{k} \lim _{n}$ relations, $g_{n k}, n<k$, may be defined indifferently.

According to Theorems 1 and 2, if

$$
\lim _{k} \lim _{n} \frac{t_{n k}}{s_{n}}=1
$$

$$
\operatorname{plim}_{\boldsymbol{k}} \operatorname{plim}_{n} h_{n k}=0 \text {, }
$$

$$
\lim _{k} \lim _{n} P\left(\frac{g_{n k}}{t_{n k}} \leqq x\right)=\frac{1}{(2 \pi)^{1 / 2}} \int_{-\infty}^{x} e^{-t^{2} / 2} d t,
$$

then

$$
\lim _{n} P\left(\frac{x_{1}+\cdots+x_{n}}{s_{n}} \leqq x\right)=\frac{1}{(2 \pi)^{1 / 2}} \int_{-\infty}^{x} e^{-t^{2} / 2} d t .
$$

The following theorems give conditions which imply (8), (9), and (10).

Theorem 3. If there exist constants $\alpha>2, B>0$ such that

$$
n / s_{n}^{2}<B, \quad n=1,2, \cdots,
$$




$$
\begin{gathered}
E\left(x_{n}^{2}\right)<B, \\
\lim _{n} \frac{\left(\sum_{1}^{n} E\left(\left|x_{i}\right|^{\alpha}\right)\right)^{1 / \alpha}}{s_{n}}=0,
\end{gathered}
$$$$
n=1,2, \cdots,
$$

then condition (11) holds.

We first establish (8) and (9): One readily finds, for $2 m<k \leqq n$,

$$
\left|s_{n}^{2}-t_{n k}^{2}\right|<\left(\left[\frac{n}{k}\right]+k^{2}\right) 8 m^{2} B
$$

and

$$
E\left(h_{n k}^{2}\right)<\frac{1}{s_{n}^{2}}\left(\left[\frac{n}{k}\right]+k^{2}\right) 8 m^{2} B .
$$

But, using (12),

$$
\begin{aligned}
\lim _{\boldsymbol{k}} \lim _{n} \frac{[n / k]+k^{2}}{s_{n}^{2}} & =\lim _{k} \lim _{n} \frac{[n / k]}{s_{n}^{2}} \\
& =\lim _{k}\left(\frac{1}{k} \lim _{n} \sup \frac{n}{s_{n}^{2}}\right)=0 .
\end{aligned}
$$

Relations (15), (16), and (17) imply (8) and (9).

Condition (10) will be true, by Liapounoff's Theorem [4, p. 284], if, for large $k$,

$$
\lim _{n} \frac{\left(\sum_{i=1}^{[n / k]} E\left(\left|y_{i}\right| \alpha\right)\right)^{1 / \alpha}}{t_{n k}}=0
$$

Now

$$
E\left(\left|y_{i}\right|^{\alpha}\right) \leqq k^{\alpha} \sum_{j=i k-k+1}^{i k-m} E\left(\left|x_{j}\right|^{\alpha}\right)
$$

so that

$$
\lim _{n} \frac{\left(\sum_{i=1}^{[n / k]} E\left(\left|y_{i}\right|^{\alpha}\right)\right)^{1 / \alpha}}{t_{n k}} \leqq \lim _{n} \frac{k\left(\sum_{i=1}^{n} E\left(\left|x_{i}\right|^{\alpha}\right)\right)^{1 / \alpha}}{s_{n}} \cdot \frac{s_{n}}{t_{n k}}=0
$$

by (14) and (8), if $k$ is large. 
TheOREM 4. If $x_{1}, x_{2}, \cdots$ is a stationary m-dependent sequence with zero means and finite variances, then (11) holds.

For in that case, (12) holds, and, since the variances are bounded, (8) and (9) are established as above. (10) holds, since, for each $k>2 m$, the sequence $y_{1}, y_{2}, \cdots$ is stationary and independent.

\section{REFERENCES}

1. H. Cramér, Mathematical methods of statistics, Princeton, 1946.

2. P. H. Diananda, Some probability limit theorems with statistical applications, Proc. Cambridge Philos. Soc. (1953) pp. 239-246.

3. W. Hoeffding and H. E. Robbins, The central limit theorem for dependent random variables, Duke Math. J. (1948) pp. 773-780.

4. J. U. Uspensky, Introduction to mathematical probability, McGraw-Hill, 1937.

University of North Carolina 